\title{
Happy talk: Perceptual and acoustic effects of smiling on speech
}

\author{
V. C. TARTTER \\ Camden College of Arts and Sciences, Rutgers University, Camden, New Jersey 08102 \\ and Bell Laboratories, Murray Hill, New Jersey 07974
}

\begin{abstract}
Smiling is a universally recognized visible expression of happiness. A side effect of smiling is an alteration of the vocal tract, suggesting that during vocalization smiling may be heard as well as seen. The present studies investigated this hypothesis. Smiled and straight-faced tokens of 29 utterances were collected from six speakers, and presented to three groups of 12 subjects for forced-choice identification. Groups 1 and 2, instructed to select the smiled or happier sound, respectively, performed significantly better than chance for all speakers. Group 3, asked to select the sadder sound, chose the straight-faced token reliably for four speakers, but for one also picked the smiled token. Acoustic analyses showed that smiling raised the fundamental and formant frequencies for all speakers and amplitude and/or duration for some. Particular cue combinations appear to be heard as smiling specifically, whereas others are associated with emotionality in general.
\end{abstract}

Smiling is a universal, innate (Dearborn, 1900; Eibl-Eibesfeldt, 1972) facial expression, most often signifying approachability (van Hooff, 1972; Mussen, Conger, \& Kagan, 1974) and pleasure (Dearborn, 1900; Shor, 1978). Frequently, smiling is accompanied by vocalization, as in smiling while greeting, smiling while complimenting, or an infant's smiling while contentedly gurgling. A side effect of smiling is alteration of the vocal tract from its neutral position: the mouth orifice widens, shortening the vocal tract, and its opening enlarges (Shor, 1978). Since some phonetic distinctions, such as lip-rounding, are produced with no greater a vocal tract change, it seems likely that the effects of smiling are audible. The present studies investigated this possibility.

If smiling can be detected and identified aurally, listeners would have an indication of the speaker's emotional state from his voice alone. Previous investigations of emotional tone have dealt exclusively with negative emotions such as emotional stress (Hecker, Stevens, von Bismarck, \& Williams, 1968; Williams, \& Stevens, 1969; Streeter, Macdonald, Apple, Krauss, \& Galotti, Note 1), deception (Friedhoff Alpert, \& Kurtzberg, 1964; Ekman, Friesen, \& Scherer, 1976; Streeter, Krauss, Geller, Olson, \&

I thank Nancy Carolyn and John Lin for their assistance in making tapes, and Lynn Streeter, Sande Pruzansky, Nina Macdonald, and James Goodnow for their suggestions. I am especially grateful to Tanya Mollenauer for her invaluable assistance, and to Debbie Flanagan for preparing the manuscript. Finally, I thank David Noreen, Osamu Fujimura, and George Sperling for their comments on an early draft. A portion of the research described in this paper was presented at the 97th meeting of the Acoustical Society of America, in Cambridge, Massachusetts, in June 1979.
Apple, 1977), sorrow and anger (Williams \& Stevens, 1972), and contempt, fear, grief, and indifference (Fairbanks \& Hoaglin, 1941; Fairbanks \& Pronovost, 1939). Among the acoustic parameters studied, pitch and pitch range show the greatest emotion-dependent fluctuations and seem to be useful in listener's identification of the emotion: pitch and pitch range increase in anger, fear, and contempt, and decrease relative to normal in less active emotions like sorrow, grief, and indifference. Duration, speech rate, amplitude, and amplitude contour have been studied sporadically. The available results suggest that amplitude and amplitude contour vary slightly, in the same direction as pitch, and duration decreases while speech rate increases for the active emotions, and conversely for the less active emotions. These parameters, too, appear to affect listeners' attributions to the speaker (Apple, Streeter, \& Krauss, in press; Fairbanks \& Hoaglin, 1941; Scherer, 1974).

The studies of emotional tone have been motivated in part by the belief that the involuntary physiological manifestations of emotion (e.g., increased respiration rate with concomitant increase in subglottal pressure, and dryness of the mouth) affect the speech signal (Williams \& Stevens, 1972). The same hypothesis may or may not apply to the emotional states underlying smiling, but the production of the smile itself, in altering the vocal tract, should have involuntary effects on the speech signal.

\section{METHOD}

To assess the effects of smiling on a range of vocal tract configurations, 29 different utterances were used. They are shown on Table 1 . There were 25 nonsense syllables, a number of consonants in the context [Ca], and the Peterson and Barney (1952) 
Table 1

Speech Types Pronounced Straight-Faced and with "Lips Spread in a Smile"

\begin{tabular}{llll}
\hline HEED & BAH & FAH & WOULD YOU LIKE SOME COFFEE? \\
HID & DAH & VAH & DID YOU HAVE A GOOD TRIP? \\
HEAD & GAH & THAH & IT'S NICE TO BE BACK HERE. \\
HAD & PAH & THAH & \\
HOD & TAH & SAH & \\
HEARD & KAH & ZAH & \\
WHO'D & MAH & SHAH & \\
HOOD & NAH & CHAH & \\
HAWED & &
\end{tabular}

syllables, differing in the medial vowel. In addition, four sentences were included, arbitrarily selected as appropriate for pronunciation either straight-faced or smiling. Three male and three female practiced informants recorded the 29 speech types, straight-faced and smiling. Each speaker was specifically instructed not to attempt to sound happy in the smiled versions, to avoid any idiosyncratic effects of acting. Since speakers reported muscle fatigue, it is unlikely that feelings of happiness were inadvertently communicated. From the utterances of each speaker, a forced-choice identification tape was prepared using a computerized speech-editing system (Nakatani, 1977). Each tape consisted of 10 trials of each of the 29 speech types, in random order. A trial was a presentation of the smiled token and its straightfaced counterpart separated by $500 \mathrm{msec}$ of silence.

Three groups of 12 local high school and college students participated in the experiment. Subjects in Condition 1 were told how the stimuli were produced and asked to select the one which sounded "smiled." The subjects in Condition 2 were instructed, instead, to select the stimulus which sounded "more cheerful" or "happier," and were not told specifically what differentiated the stimuli. This was to see if listeners would identify the effects of smiling with the emotions normally evoking smiling, without knowing what to listen for. Likewise, subjects in Condition 3 were not told specifically about the stimuli; however, they were asked to select the "sadder"-sounding member of the pair. This was a control to determine if listeners were actually identifying the specific emotion or if they were picking the stimulus which sounded more deviant or more emotional.

All subjects listened to all six tapes, three on one day and three the following. Half the subjects in each condition listened to the tapes in the same order, the other half, in the reverse order. The subjects were tested in small groups, in a soundproofed room, with the stimuli presented over earphones. No familiarization with the stimuli was given beyond the practice trials. The subjects had a short break between tapes. Each experimental session lasted approximately $1 \frac{1}{2} \mathrm{~h}$.

\section{RESULTS AND DISCUSSION}

Results for the three conditions are displayed in Table 2. For each condition, Column 1 shows the percentage of smiled tokens selected, averaged over the 12 subjects, and Column 2 shows the results of a two-tailed t test comparing actual performance to chance. Two results are apparent from examination of just the data from the "smile" condition: First, and most important, subjects identified the smiled sound significantly more often than chance for all speakers; and second, for some speakers it was easier to identify smiling than for others. A simple withinsubjects analysis of variance showed that the speaker differences were reliable $[F(5,55)=8.96, p<.001]$, and post hoc comparisons indicated that this was due entirely to better performance for speakers $M$.W. and M.M. $(\mathrm{p}<.05)$.

Examination of the results for the "happy" and "sad" conditions indicates that the smiled and straight-faced tokens were identified, respectively, with happier and sadder emotions. Comparison of the percentage correct for different speakers shows large speaker effects in these conditions too $[F(5,55)$ $=7.92, \mathrm{p}<.001$, for "happy"; $F(5,55)=35.51$, $\mathrm{p}<.01$, for "sad"']. Subjects in the "happy" condition identified smiled sounds with happiness significantly more often in speaker M.W. and less often in speakers P.T. and R.K. than in the other speakers $(\mathrm{p}<.05)$.

Subjects in the "sad" condition performed significantly differently on speakers M.M. and P.T. than on all the other speakers: M.M.'s smiled speech was identified as "sad" reliably, and labeling of P.T.'s speech was random. A within-between analysis of variance contrasting performance across the three conditions showed significant effects of Instructions $[F(2,33)=8.84, p<.01]$, Speakers $[F(5,165)=$ $23.65, \mathrm{p}<.01]$, and their interaction $[\mathrm{F}(10,165)=$

Table 2

Forced-Choice Identification for the 12 Subjects in Each Instruction Condition

\begin{tabular}{|c|c|c|c|c|c|c|c|c|c|}
\hline \multirow[b]{2}{*}{ Speaker } & \multicolumn{3}{|c|}{ Smile } & \multicolumn{3}{|c|}{ Happy } & \multicolumn{3}{|c|}{ Sad } \\
\hline & PC & $\mathrm{t}$ & $\mathrm{p}$ & $\mathrm{PC}$ & $t$ & p & PC & $\mathrm{t}$ & $\mathrm{p}$ \\
\hline P.T. & 64.6 & -3.18 & .01 & 58.7 & -2.28 & .05 & 49.6 & .12 & $.05^{*}$ \\
\hline V.T. & 65.7 & -5.70 & .002 & 61.1 & -3.04 & .02 & 62.7 & -2.96 & .05 \\
\hline K.M. & 69.8 & -6.66 & .002 & 67.7 & -4.04 & .002 & 57.7 & -2.43 & .05 \\
\hline M.W. & 79.3 & -5.94 & .002 & 76.5 & -7.18 & .002 & 77.8 & -6.50 & .02 \\
\hline R.K. & 63.0 & -4.54 & .002 & 52.8 & -1.74 & .1 & 58.0 & -2.90 & .05 \\
\hline M.M. & 82.2 & -9.68 & .002 & 63.6 & -4.27 & .02 & 27.4 & 6.21 & .002 \\
\hline
\end{tabular}

Note-For "smile" and "happy," percent correct (PC) reflects the average percentage of smiled tokens selected, for "sad," the average percentage of straight-faced tokens selected. The t tests contrasted subjects' performances to chance, $50 \%$ or 145 correct. ${ }^{*} p>.05$ in this case: in all other cases, $p<$ the value given. 
$14.31, \mathrm{p}<.011$. Post hoc comparisons indicated that the "sad" instructions were harder than either of the others, that overall M.W. was the easiest speaker for whom to label emotions and K.M. and V.T. next easiest, and that performance was affected by instructions for only two of the six speakers, P.T. and M.M.

There are three important conclusions to be drawn from these results. First, clearly smiling has an audible effect on speech. Second, in most instances, these audible effects are automatically associated with the positive emotions that normally give rise to smiling, rather than with emotionality in general. Third, listeners appear to have precise and consistent criteria for what contrasts happy and sad tones of voice. Four speakers unambiguously met these criteria; P.T.'s smiled speech sounded smiled and happy, but his neutral speech in comparison did not sound sad; and M.M.'s smiled speech sounded deviant and not clearly happy or sad. In this regard, note also that results for "sad" overall were poorer. This may be because straight-faced tokens are not necessarily sad, but only so in comparison with smiled speech.

If smiling produces audible effects on speech, there must be acoustic correlates in the signal. Specification of these acoustic properties not only is informative as to the cues for smiling, but more generally provides important data for acoustic theory on the effects of one extreme vocal tract configuration (cf. Ladefoged, Wright, \& Linker, Note 2 , for data on the other extreme). In addition, in this experiment in particular, acoustic differences in smiled speech between speakers can be related to the perceptual criteria for happy or sad sounding voices.

Digital spectrograms were made of the 25 syllables from which measurements of the steady state formant frequencies of the first three formants were derived. In addition, the stimuli were LPC-analyzed with pitch extraction (Atal \& Hanauer, 1971), and from these analyses average fundamental frequency, amplitude, and duration were computed for each token. Table 3 summarizes the results of these computations, together with results of correlated t tests comparing the smiled and straight-faced values for each speaker.

Note first the pattern of results for V.T., K.M., and M.W., the speakers most consistently judged in the threc conditions. For all, formant frequencies increased substantially when smiling. Moreover, fundamental frequency, the parameter found to be most salient in the earlier studies of perceived emotion, increased consistently. Amplitude also was greater for these speakers (though not significantly for K.M.). There was no reliable change in duration. Note further that the frequency and amplitude increases were greatest for M.W., whose speech was easiest to label in the three conditions.

The three speakers remaining, for whom there was some confusion, contrast with the others in at least one of these parameters. P.T. and R.K., the more difficult speakers in "smile" and "happy," are the only two for whom there was no reliable pitch increase. Further, P.T.'s amplitude dropped during smiling, and this lower amplitude, associated with less active emotions in earlier studies, may have been the conflicting information resulting in the random responding in the "sad" condition. For M.M., although there were substantial fundamental and formant frequency increases, there was no change in amplitude and a highly significant increase in syllable duration, which was likely to be associated with the slowed rate of sadder emotions. The marked frequency and duration changes in all likelihood resulted in her smiled speech being perceived as emotional, but the quality of the emotion was not peculiar to either stereotyped happiness or sadness. This analysis suggests incorrectly that R.K.'s smiled speech should also have been labeled "sad," since he too slowed his speech considerably. Perhaps duration is not a salient cue unless in combination with other marked acoustic parameters.

Acoustic specifications of the effects of smiling are important specifically for determining how emotion is detected in the voice, and more generally, for acoustic theory concerning the effects of the most extreme shortening of the vocal tract. With respect to the latter, there was little reason to expect either

Table 3

Summary of Acoustic Analyses of Smiled and Straight-Faced Speech for Each Speaker

\begin{tabular}{|c|c|c|c|c|c|c|}
\hline \multirow[b]{2}{*}{ Speaker } & \multicolumn{3}{|c|}{ Formant Frequencies (Hertz) } & \multirow{2}{*}{$\begin{array}{c}\text { Fundamental } \\
\text { Frequency (Hertz) }\end{array}$} & \multirow{2}{*}{$\begin{array}{c}\text { Amplitude } \\
\text { (RMS) }\end{array}$} & \multirow{2}{*}{$\begin{array}{c}\text { Duration } \\
\text { (Milliseconds) }\end{array}$} \\
\hline & $F_{1}$ & $\mathrm{~F}_{2}$ & $F_{3}$ & & & \\
\hline P.T. & $22 *$ & $76 \dagger$ & 20 & 1.25 & $-34.41 * *$ & $-46.0 * *$ \\
\hline V.T. & 14 & $68 t$ & $71+$ & $6.78 * *$ & $8.00^{* *}$ & 16.2 \\
\hline K.M. & $18^{*}$ & $44 * *$ & $84 \dagger$ & $5.57 * *$ & 5.53 & -23.1 \\
\hline M.W. & $100+$ & $110^{\circ}$ & $171+$ & $16.19 * *$ & $25.64 * *$ & 34.2 \\
\hline R.K. & 8 & $50^{* *}$ & $154 \dagger$ & 2.00 & $8.86^{*}$ & $125.6 \dagger$ \\
\hline M.M. & $26 * *$ & $86+$ & $80 \dagger$ & $14.60 * *$ & .04 & $110.0 \dagger$ \\
\hline
\end{tabular}

Note-Average differences represent the average value for the smiled token minus the average value of the straight token. Significance values were obtained from correlated tests comparing the values on the 29 utterances of each speaker.

${ }^{*} p<.05 . \quad * * p<.01, \quad t p<.002$. 
pitch or duration to be affected by the gesture of smiling. Indeed, the duration change was not necessary, since it appeared only idiosyncratically. The pitch increase may arise automatically from coupling of laryngeal tensing with facial tension. Fundamental frequency differences have been reported for different vowels (Peterson \& Barney, 1952) and may, in fact, be somewhat dependent on size and shape of the vocal tract. The principal acoustic effects smiling was expected to produce were an increased amplitude resulting from the larger mouth opening and increases in the formant frequencies resulting from the shortened vocal tract. As the data showed, these results were obtained for most speakers.

In conclusion, the results of the present studies showed that naive listeners can identify smiled speech as such and indicated what cues they may have been using. Of course, this was for isolated speech samples produced under rather controlled and unnatural conditions. Presumably, under more normal conditions, with "happier" intonations and longer samples, performance would be even better and there would be additional acoustic effects. However, even in the present case, for most speakers, smiled speech was associated with the pleasant emotions usually producing it. This suggests that people wishing to convey a positive, approachable, auditory impression, should smile while speaking. Moreover, the detailed acoustic specifications of smiled speech may enable this favorable auditory impression to be extended to computer-synthesized speech.

\section{REFERENCE NOTES}

1. Streeter, L. A., Macdonald, N. H., Apple, W., Krauss, R. M., \& Galotti, K. M. Acoustical and perceptual indicators of emotional stress. Paper presented at the 96th Meeting of the Acoustical Society of America, Honolulu, 1978.

2. Ladefoged, P., Wright, J., \& Linker, W. Where does the vacal tract end? Paper presented at the 95th Meeting of the Acoustical Society of America, Providence, Rhode Island 1978.

\section{REFERENCES}

Apple, W., Streeter, L. A., \& Krauss, R. M. The effects of pitch and speech rate on personal attributions. Journal of Personality and Social Psychology, in press.
Atal, B. S., \& Hanauer, S. C. Speech analysis and synthesis by linear prediction of the speech wave. Journal of the Acoustical Society of America, 1971, 50, 637-655.

Dearborn, G. V. N. The nature of the smile and laugh. Science, $1900,11,851-856$.

Erbl-EibesfeldT, I. Similarities and differences between cultures in expressive moments. In R. A. Hinde (Ed.), Nonverbal communication. Cambridge: Cambridge University Press, 1972.

Ekman, P., Friesen, W. V., \& Scherer, K. R. Body Movement and voice pitch in deceptive interaction. Semiotica, 1976, 16, 23-27.

Fairbanks, G., \& Pronovost, W. Ar experimental study of the pitch characteristics of the voice during the expression of emotion. Speech Monographs. 1939, 6, 89-104.

Fairbanks, G., \& Hoaglin, L. W. An experimental study of the durational characteristics of the voice during the expression of emotion. Speech Monographs, 1941, 8, 85-90.

Friedhoff, A. J., Alpert, M. \& Kurtzberg, R. L. Infracontent channels of vocal communication. Association for Research of Nervous and Mental Disease, 1964, 42, 414-423.

Hecker, M. H. L., Stevens, K. N., von Bismarck, G., \& Williams, C. E. Manifestations of task-induced stress in the acoustic speech signal. Journal of the Acoustical Society of America, 1968, 44, 993-1001.

van Hooff, J. A. R. A. M. A comparative approach to the phylogeny of laughter and smiling. In R. A. Hinde (Ed.), Nonverbal communication. Cambridge: Cambridge University Press, 1972.

Mussen, P. H., Conger, J. J., \& Kagan, J. Child development and personality (4th Ed.). New York: Harper \& Row, 1974.

Nakatani, L. H. Computer-aided signal handling for speech research. Journal of the Acoustical Society of America, 1977, 61, 1056-1062.

Peterson, G. E., \& Barney, H. L. Control methods used in a study of vowels. Journal of the Acoustical Society of America, 1952, 24, 175-184.

Scherer, K. R. Acoustic concomitants of emotional dimensions: Judging affect from synthesized tone sequences. In S. Weitz (Ed.), Nonverbal communication. New York: Oxford University Press, 1974.

SHor, R. E. The production and judgment of smile magnitude. The Journal of General Psychology, 1978, 98, 79-96.

Streeter, L. A., Krauss, R. M., Geller, V. J., Olson, C., \& APPLE, W. Pitch changes during attempted deception. Journal of Personality and Social Psychology, 1977, 35, 345-350.

Williams, C. E., \& Stevens, K. N. On determining the emotional state of pilots during flight: An exploratory study. Aerospace Medicine, 1969, 40, 1369-1372.

Williams, C. E., \& Stevens, K. N. Emotions and speech: some acoustical correlates. Journal of the Acoustical Society of America, 1972, 52, 1238-1250.

(Received for publication July 16, 1979; revision accepted November 8,1979 .) 